\title{
CROSSOVER OF THE COARSENING RATES IN DEMIXING OF BINARY VISCOUS LIQUIDS*
}

\author{
FELIX OTTO ${ }^{\dagger}$, CHRISTIAN SEIS ${ }^{\ddagger}$, AND DEJAN SLEPČEV $§$
}

\begin{abstract}
We consider a model for phase separation in binary viscous liquids that allows for material transport due to cross-diffusion of unlike particles and convection by the hydrodynamic bulk flow. Typically, during the evolution, the average size of domains of the pure phases increases with time - a phenomenon called coarsening. Siggia [Eric D. Siggia, Phys. Rev. A, 20(2), 595-605, Aug. 1979] predicts that at an initial stage, coarsening proceeds mainly by diffusion, which leads to the well-known evaporation-recondensation growth law $\ell \sim t^{1 / 3}$, when $\ell$ denotes the average domains size and $t$ denotes time. Furthermore, he argued that at a later stage, convection by the bulk flow becomes the dominant transport mechanism, leading to a crossover in the coarsening rates to $\ell \sim t$. Siggia's predictions have been confirmed by experiments and numerical simulations.

In this work, we prove the crossover in the coarsening rates in terms of time-averaged lower bounds on the energy, which scales like an inverse length. We use a method proposed by Kohn and the first author [Robert V. Kohn and Felix Otto, Commun. Math. Phys., 229(3), 375-395, 2002], which exploits the gradient flow structure of the dynamics. Our adaption uses techniques from optimal transportation. Our main ingredient is a dissipation inequality. It measures how the optimal transportation distance changes under the effects of convective and diffusive transport.
\end{abstract}

Key words. Coarsening, demixing, phase segregation.

AMS subject classifications. 35K55, 82C24, 35Q35, 35G20, 82D80, 76D07, 35B99.

\section{Introduction}

We investigate the coarsening rates in the demixing process of binary viscous liquids. Demixing in liquid mixtures typically arises when the mixture is put into a thermodynamically unstable state far from an equilibrium, for instance, after a quench from a high to a sufficiently low temperature. Thermodynamics favors the separation of the two phases. This drives the formation of microstructure: Initially, the mixture demixes locally by forming intertwined domains of the two pure phases. The free energy concentrates along the interface between these domains. In the subsequent evolution, the system tends to equilibrium by reducing the interfacial area. During this coarsening process the length scales describing the configuration (such as the average domain width) grow.

In binary viscous liquids, there are two parallel transport mechanisms. Material is transported by cross-diffusion, the relative motion of the two different species through the bulk, and by convection, the material transport by the bulk flow. It turns out that each transport mechanism becomes dominant during a certain time interval in the demixing process. Initially, demixing is mediated mainly by diffusion. Later on, as the domains become large enough, viscous forces in the liquid become effective and the flow transport becomes the more efficient mechanism.

We are interested in the rate at which the coarsening of the domain morphology proceeds. Generically, the system develops a typical length scale $\ell$ that describes the typical width of the domains of the pure phases. Therefore, the coarsening rate is

\footnotetext{
*Received: May 2, 2012; accepted: June 22, 2012. Communicated by Chun Liu.

${ }^{\dagger}$ Max-Planck-Institut für Mathematik in den Naturwissenschaften, Inselstr. 22, 04103 Leipzig, Germany (otto@mis.mpg.de).

${ }^{\ddagger}$ Department of Mathematics, University of Toronto, 40 St. George St., Toronto, Ontario, Canada M5S 2E4 (cseis@math.toronto.edu).

$\S$ Department of Mathematical Sciences, Carnegie Mellon University, 5000 Forbes ave., Pittsburgh, PA 15213, USA (slepcev@math.cmu.edu).
} 
measured by the growth rate of $\ell$. In 1979, in a seminal paper [22], Siggia argued that during the first, diffusion-dominated regime, the coarsening rate behaves like $\ell \sim t^{1 / 3}$ — the well-known coarsening law from the evaporation-recondensation process (Ostwald ripening) studied by Lifshitz, Slyozov, and Wagner [14, 29]. Siggia predicted that at a later stage, the dominance of convection leads to a crossover of the coarsening rate to $\ell \sim t$. Siggia's coarsening rates have been confirmed in numerical simulations 2, 11, 20, 25, and physical experiments [6, 30, 31, 13, see also the discussion in Section 3

In this paper, we rigorously establish Siggia's coarsening rates in the form of weak upper bounds: We show that coarsening cannot proceed faster than $\ell \lesssim t^{1 / 3}$ for diffusion-mediated and subsequently $\ell \lesssim t$ for convection-mediated transport. The coarsening rates come in the form of time-averaged lower bounds on the energy density; for a discussion on the energy scaling, see page 447.

Our mathematical investigation of the crossover of the coarsening rates fuses together the individual results on coarsening rates for purely diffusive transport [12] and for purely convective transport 4. We apply the general method introduced in 12. There are two questions that arise. One concerns the technique:

Can the method of [12] be adapted to capture the crossover of coarsening rates which reflect two different transport regimes?

The second issue is more fundamentally related to the nature of the system:

Can the bulk transport mechanism sufficiently "help" the diffusive transport during the stage when the later is dominant, for the coarsening rate to be affected (and vice versa)?

Below we answer the first question affirmatively and the second negatively.

Recently, Dai, Niethammer, and Pego [8 obtained rigorous results on the crossover of coarsening rates from attachment-limited to diffusion-limited dynamics as it occurs in rapid solidification of melts. They too used the framework of [12. In addition to being a different system and a mean-field-type approximation (we work with a phase-field model), a significant difference from our work here is that in [ 8 , the system features two limiting dissipation mechanisms rather than two transport mechanisms. The authors prove the crossover from $\ell \sim t^{1 / 2}$ in the regime of attachment dynamics to $\ell \sim t^{1 / 3}$ in the regime of diffusion dynamics. The presence of two limiting dissipation mechanisms slows down the coarsening process, $\ell \sim \min \left\{t^{1 / 2}, t^{1 / 3}\right\}$, while the presence of two transport mechanisms speeds it up, $\ell \sim \max \left\{t^{1 / 3}, t\right\}$.

The paper is organized as follows: In Section 2 we introduce the model, both in the diffuse-interface and the sharp-interface formulation. In Section 3 we present a heuristic argument for the rates of coarsening and determine at what length scale should the crossover occur. In Section 4 we state the main result. We present the technique used and state the propositions that combine to establish the main result. In Section 5 we present several results on how the optimal transportation distance changes under the effects of (convective and diffusive) transport. These results are needed to establish the propositions of Section 4, but are of independent interest as well. In Section 6 we present the detailed proofs of the statements of Section 4. Finally in Appendix A, we prove an optimal-transportation result that we need. Let us also mention that an extended version of the paper which contains a discussion on well-posedness and regularity (in three space dimensions) of the system of PDE describing the demixing process is available both as a CNA preprint and as an MPI preprint [18. 


\section{The models}

We present our results in detail for the diffuse-interface model. The dynamics are described in terms of an order parameter (or phase field), which measures the local composition of the mixture. The order parameter varies smoothly between the pure phases, as is expected in diffusive systems. We also introduce the sharp-interface model, which depicts the thermodynamics on a mesoscopic level. As the name suggests, the diffuse interface is replaced by a sharp borderline between the domains of the pure phases, where the order parameter is supposed to be constant. This approximation is justifiable in many systems if the typical domain size is much larger than the interfacial width; see for example [19, 3, 5, 1]. While for the diffuse-interface model the questions of well-posedness and regularity can be answered by standard techniques, the well-posedness theory for the sharp-interface model remains open. Nevertheless, since the sharp-interface system allows for a clear heuristic argument for the crossover of the rate of coarsening, we introduce it and briefly comment on the results on the coarsening of that system as well.

We consider the problem in a periodic setting. Let $\Omega \subset \mathbb{R}^{d}, d \geq 2$, be the cell of periodicity of all functions considered. We assume that

$$
|\Omega| \gg 1 \text {. }
$$

Since we are always working with averages, the system size, i.e., the volume of $\Omega$, will not enter in our analysis. For any measurable set $A \subset \Omega$, we write $f_{A}=\frac{1}{|\Omega|} \int_{A}$ for convenience.

The mathematical quantity that models the physical configuration is the order parameter $m$, which describes the local composition of the mixture. It takes the values \pm 1 in the stable phases. For symmetry reasons, $m$ takes value zero where both phases are mixed in equal measure. Since we exclude chemical reactions, the total mass of each phase is conserved during the evolution. We consider the critical mixture, where both phases occupy the same volume fraction, so that

$$
\bar{m}:=f_{\Omega} m d x=0 .
$$

The case $\bar{m} \neq 0$ can be treated analogously.

Below we introduce the models. For a more detailed introduction of the models as well as an explanation of their gradient-flow structure, we refer to 4 .

2.1. Diffuse-interface model. We begin with the introduction of the model for diffuse interfaces. The free energy, averaged over cell $\Omega$, is given by the GinzburgLandau functional with the classical double-well potential:

$$
E(m):=f_{\Omega} \frac{1}{2}|\nabla m|^{2}+\frac{1}{2}\left(1-m^{2}\right)^{2} d x .
$$

It favors the values $m=1$ and $m=-1$, representing the pure phases, and penalizes transitions between these values. It is nondimensionalized in such a way that the typical transition layer between the domains of the pure phases is of order one, and thus much smaller than the system size. The $L^{2}$ derivative of the energy is the chemical potential

$$
\mu:=\frac{\partial E}{\partial m}=-\Delta m-2\left(1-m^{2}\right) m
$$


Since the order parameter must be conserved, the evolution equation for $m$ comes in the form of a local conservation law, $\partial_{t} m+\nabla \cdot J=0$. Due to the two parallel transport mechanisms, the transporting flux combines diffusion and convection, $J=\lambda J_{\text {diff }}+J_{\text {conv }}$, where $\lambda$ measures the relative strength of the diffusion flux compared to the convection flux. The flux due to cross-diffusion is given by $J_{\text {diff }}=-\nabla \mu$, and the flux due to fluid convection is $J_{\text {conv }}=m u$, where $u$ denotes the fluid velocity. It obeys a Stokes equation:

$$
\begin{aligned}
-\Delta u+\nabla p & =-m \nabla \mu, \\
\nabla \cdot u & =0,
\end{aligned}
$$

in which $p$ is the hydrodynamic pressure. The forcing term which acts on $u$ is determined by the "principle of virtual work": It guarantees that the dissipation due to convection is given by the Stokes friction term (cf. (2.5)). Summing up, the demixing process is described by the equation

$$
\partial_{t} m-\lambda \Delta \mu+\nabla \cdot(m u)=0
$$

where $\mu$ is given by (2.2) and $u$ solves the Stokes Equation (2.3). This model is nondimensionalized and permits - besides the size of the system - one dimensionless parameter: $\lambda$. Well-posedness and smoothness of solutions, for the physically relevant dimensions $d=2,3$, can be established using the standard energy based methods. We do not present them in this paper. However an extended version of the paper that contains the proofs of these results is available [18.

Direct calculation shows that the energy dissipation rate is

$$
\frac{d E}{d t}=-f_{\Omega} \lambda|\nabla \mu|^{2}+|D u|^{2} d x
$$

2.2. Sharp-interface model. On the mesoscopic level, $m$ takes only the values which characterize the pure phases, i.e., $m= \pm 1$. The energy concentrates on the interface and is proportional to its area (length in two space dimensions). A straightforward computation of the energy of the one-dimensional interfacial profile determines the prefactor $\frac{4}{3}$. We thus define

$$
E(m):=\frac{4}{3} \times \frac{1}{2} f_{\Omega}|\nabla m| d x=\frac{2}{3} f_{\Omega}|\nabla m| d x .
$$

Let $\Omega_{+}$be the subset of $\Omega$ where $m$ takes value 1 , and $\Omega_{-}$the subset where $m=-1$. Let $\Gamma=\partial \Omega_{+}$. We denote the mean curvature of $\Gamma$ by $H$, with the convention that $H$ is nonnegative along convex boundaries $\partial \Omega_{+}$.

The chemical potential $\mu$ is given by

$$
\begin{aligned}
-\Delta \mu & =0 & & \text { in } \Omega \backslash \Gamma, \\
\mu & =\frac{2}{3} H & & \text { on } \Gamma .
\end{aligned}
$$

The fluid velocity $u$ solves the following Stokes equation in which $S=\left(D u+(D u)^{T}\right)-$ $p I$ is the stress tensor, $p$ is the hydrodynamic pressure, $\tau$ is any tangent vector to $\Gamma$, 
and $\nu$ is the outside (relative to $\Omega_{+}$) unit normal vector to $\Gamma$ :

$$
\begin{aligned}
-\nabla \cdot S & =0 & & \text { in } \Omega \backslash \Gamma, \\
\nabla \cdot u & =0 & & \text { in } \Omega, \\
\tau[S \nu] & =0 & & \text { on } \Gamma, \\
\nu[S \nu] & =-\frac{4}{3} H & & \text { on } \Gamma .
\end{aligned}
$$

In the above system, $[A]$ denotes the jump in quantity $A$ across $\Gamma$.

Again, the order parameter $m$ is transported by the total flux. That is, the interface $\Gamma_{t}$ moves with normal velocity

$$
V=-\frac{1}{2}\left[\frac{\partial \mu}{\partial \nu}\right]+u \cdot \nu
$$

While the second term in the velocity is just the transport by the bulk flow, the first term is well known from the Mullins-Sekerka law. Notice that this sharp interface version of (2.3) \& (2.4) is - besides the system size - free of dimensionless parameters. This comes from the fact that there is no length scale that corresponds to interfacial width. Thus, in any statements in which $\lambda$ appears and that apply to sharp interfaces, one should set $\lambda=1$.

\section{Heuristics}

In this section, we present a simple heuristic argument in favor of the two coarsening rates. A fairly neutral starting point for demixing studies is an almost uniform state corresponding to full mixing, say $m \approx 0$. This configuration is unstable and linear analysis and numerical simulations (cf. 4) indicate that a wavelength of order one grows the fastest. A natural assumption on the typical domain size $\ell$ at the onset of coarsening is therefore

$$
\ell \gtrsim 1
$$

It is expected, as such behavior is ubiquitous in related energy-driven systems, that in large systems, the evolution of the characteristic length scale obeys a power law:

$$
\ell(t) \sim t^{\gamma} .
$$

The coarsening exponent $\gamma$ is determined by the dominant material transport mechanism. We argue that $\gamma=1 / 3$ for diffusion-mediated and $\gamma=1$ for convection-mediated transport.

The heuristics are based on the assumption that the coarsening evolves statistically self-similarly in the interfacial regime. This means

$$
|\mathcal{F} m(t, \cdot)(k)|^{2} \approx f\left(t^{\gamma} k\right)
$$

for some structure function $f$ and every wave number $k$. Above, $\mathcal{F} m$ denotes the Fourier transform of $m$, i.e., $\mathcal{F} m(k)=f_{\Omega} m(x) e^{i k \cdot x} d x$. Such a behavior is suggested by numerical simulations, but, to our knowledge, there are no rigorous results in this direction.

In order to determine the different coarsening exponents, we treat the underlying dominant transport mechanisms separately. We consider the sharp-interface models. 
In the purely diffusive system, the evolution of the interface $\Gamma$ is the Mullins-Sekerka law

$$
V=-\frac{1}{2}\left[\frac{\partial \mu}{\partial \nu}\right] \quad \text { on } \Gamma
$$

where $\mu$ is the chemical potential defined via (2.7). Solutions are invariant under the scaling

$$
x=\alpha \hat{x}, \quad t=\alpha^{3} \hat{t}
$$

and therefore, assuming statistical self-similarity,

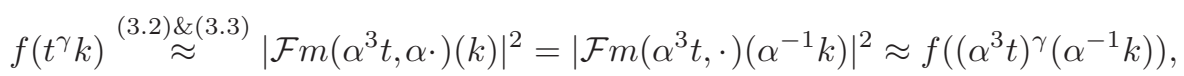

which yields $\gamma=1 / 3$, since $\alpha$ is arbitrary. Thus

$$
\ell(t) \sim(\lambda t)^{1 / 3}
$$

To illustrate the $\lambda$-dependence of the coarsening rate, observe that in the purely diffusive version of (2.4), $\lambda$ can be absorbed into the time scale via the transformation $\hat{t}=\lambda t$.

In the purely convective system, the evolution of the interface $\Gamma$ is

$$
V=u \cdot \nu
$$

where $u$ solves (2.8). Solutions are invariant under the scaling

$$
x=\alpha \hat{x}, \quad t=\alpha \hat{t} .
$$

This implies, assuming statistical self-similarity, by a similar computation as above,

$$
\ell(t) \sim t
$$

Comparing both coarsening rates, we expect the crossover from the diffusion-dominated to the convection-dominated regime at time and length, respectively, to be

$$
t_{\text {crossover }} \sim \lambda^{1 / 2}, \quad \ell_{\text {crossover }} \sim \lambda^{1 / 2} .
$$

In particular, in view of (3.1), we may think of $\lambda \geq 1$ in order to treat a crossover situation.

The heuristic argument presented in this section and the rigorous results in the remainder of this paper apply in any space dimension larger than or equal to two, in particular in the physically important case of three dimensions. However, in dimension two, the validity of the convection-dominated growth law is under controversy in the physics literature. There are basically two positions. On the one hand, in [21, the authors claim that in two dimensions, the curvature-driven Stokes flow induced by (2.8) does not yield the appropriate evolution law of the interface, and therefore, the linear growth law $\ell \sim t$ does not hold. The argument is based on the investigation of Rayleigh instabilities in two dimensional hydrodynamic fluids; linear stability analysis indicates that long cylindrical tubes of fluid surrounded by fluid of different density are stable under long wavelength perturbations - as opposed to the situation in three 
dimensions. Therefore, the authors deduce that convective coarsening is not possible. It is suggested that in two dimensions the linear coarsening rate is replaced by $\ell \sim t^{1 / 2}$ corresponding to droplet coalescence. This scaling law has also been numerically observed [26, 15]. On the other hand, the author of [9] explains the discrepancy of the coarsening rates in two and three dimensions by the occurrence of many isolated spherical domains in two dimensions, for which the hydrodynamic bulk flow is not effective, and which coarsen therefore according to the evaporation-recondensation growth law $\ell \sim t^{1 / 3}$. This thesis is supported by the numerical computation of different measures of length scales (different moments of the structure function). As a particular consequence, in two dimensions, the presence of a sole characteristic length scale is questionable (the largest domains, however, still show linear coarsening behavior). In three dimensions, one observes a rich connectivity among the domains, so that the accumulation of isolated droplets is less apparent. Despite this unsatisfactory ambiguity in the state of the art in 2- $d$ coarsening of binary viscous fluids, the present work contributes to the discussion, proving that, even in two space dimensions, coarsening cannot proceed faster than $\ell \sim t$ in the convective regime (more precisely, we show that the interfacial area cannot decrease too fast).

\section{Results and method}

Let us rigorously state our main results. We focus on the diffuse-interface setting, that is, we consider the energy density defined in (2.1), and the (gradient flow) dynamics given by (2.3) \& (2.4). When it might be of interest, we also shortly comment on the corresponding sharp-interface versions of our results.

Our main result, Theorem 4.1 below, describes a crossover in the coarsening rates from $\ell \sim(\lambda t)^{1 / 3}$ in a diffusion dominated regime to $\ell \sim t$ in a convection dominated regime. The result comes in the form of time-averaged lower bounds on the energy density (see the discussion on page 448). Before presenting Theorem 4.1, we still need some preparation.

Our analysis uses two different notions of intrinsic length scales, a geometric one and a physical one. The canonical candidate for a geometric length scale is the inverse energy density of the system, which, because

$$
E \approx \text { energy of one-dimensional interfacial layer } \times \frac{\text { area of interface }}{\text { volume of system }}
$$

(cf. (2.6)), indeed scales like a length (heuristically at least). The physical length scale is defined via a transportation distance, which we call a Monge-KantorovichRubinstein distance (MKR distance) in its most abstract form, and which will be introduced presently.

For any two measures $\theta, \vartheta$ on $\Omega$, with $\theta(\Omega)=\vartheta(\Omega)$ the MKR distance $d_{c}$ with cost $c$ is defined by

$$
d_{c}(\theta, \vartheta):=\inf _{\pi \in \Pi(\theta, \vartheta)} f f_{\Omega \times \Omega} c(x, y) d \pi(x, y),
$$

where $\Pi(\theta, \vartheta)$ is the set of all couplings between $\theta$ and $\vartheta$, that is, the set of all measures $\pi$ on $\Omega \times \Omega$ such that

$$
\pi(A \times \Omega)=\theta(A) \quad \text { and } \quad \pi(\Omega \times B)=\vartheta(B) \text { for all measurable sets } A, B .
$$

Here, we use the notation $\int f_{A \times B}=\frac{1}{|\Omega|} \iint_{A \times B}$ for any measurable sets $A$ and $B$. 
With the cost function $c$ defined by

$$
c(z):= \begin{cases}\lambda^{-1 / 2} z & 0 \leq z \leq \lambda^{1 / 2} \\ 1+\ln z-\ln \lambda^{1 / 2} & \lambda^{1 / 2} \leq z\end{cases}
$$

and

$$
m_{+}:=\max \{m, 0\} \quad \text { and } \quad m_{-}:=\max \{-m, 0\},
$$

we set

$$
L:=d_{c}\left(m_{+}, m_{-}\right) .
$$

As we shall explain later, the latter can be considered a physical length of the system. Let $\Omega=[0, \Lambda]^{d}$. For $z \in \mathbb{R}^{d}$ we set $\|z\|=\min \left\{\left|z+\Lambda\left(\alpha_{1} e_{1}+\cdots \alpha_{d} e_{d}\right)\right|: \alpha_{1}, \ldots, \alpha_{d} \in \mathbb{Z}\right\}$, where $e_{i}$ are unit coordinate vectors. Note that if $\Omega$ is considered as a flat torus then the distance between $x$ and $y$ in $\Omega$ is $\|x-y\|$. We use $c(x, y)=c(\|x-y\|)$ as a symbol for both a function from $\Omega \times \Omega \rightarrow[0, \infty)$ and a function from $[0, \infty) \rightarrow[0, \infty)$ as it is clear from the argument which one is considered. Note that, since $\bar{m}=0$, we have $f_{\Omega} m_{+}(x, t) d x=f_{\Omega} m_{-}(x, t) d x$ for all $t \geq 0$. We remark that for $\bar{m} \neq 0$, one would consider $(m-\bar{m})_{+}$and $(m-\bar{m})_{-}$.

Before motivating the definition of $c$ and commenting on the interpretation of $L$ as a physical length in our dynamical system, we present our main result and discuss the analytical method we apply.

TheOREM 4.1. Let $E(m(0)) \ll 1$ and $L(m(0)) \ll 1$. Then

$$
\int_{0}^{T} \max \left\{\lambda^{1 / 2} E^{2}, E\right\} d t \gtrsim \int_{0}^{T} \min \left\{\lambda^{1 / 2} \frac{1}{(\lambda t)^{2 / 3}}, \frac{1}{t}\right\} d t
$$

for all $T$ such that $\lambda^{-1 / 2} T \gg L(m(0))^{3}$.

REMARK 4.2. The above result, with $\lambda=1$ and without any assumption on $E(m(0))$, also holds for the sharp interface system under the assumption that the sharp-interface system has smooth enough solutions.

We use the sloppy notation " $\gtrsim$ " and "》" to indicate that the inequality holds up to a generic constant which may depend on the space dimension only, provided that $E(m(0))$ and $L(m(0))$ are sufficiently small and $\frac{\lambda^{-1 / 2} T}{L(m(0))^{3}}$ is sufficiently large. In particular, the statement in Theorem 4.1 is uniform in $L(m(0))$ and $\lambda$, and we formally obtain the individual results from [12] (pure diffusion) and [4] (pure convection) in the asymptotic limits $\lambda \gg 1$ and $\lambda \ll 1$, respectively.

The result of Theorem 4.1 above is in agreement with the physical prediction in 22. Indeed, since the normalized energy scales like an inverse length (see discussion above) and assuming that there is only one length scale present in the dynamics, a lower bound on the energy can be interpreted as an upper bound on the coarsening rate. As other results based on the technique of [12, our analysis produces only timeaveraged and only lower bounds on the energy. (A counter-example for a pointwise version of the coarsening rates derived within this method is given in the original paper [12, Remark 4].) The condition on $T$ gives a bound for the minimal size of the time interval over which the coarsening rates are averaged. Also, we have no rigorous arguments to precisely determine the crossover time in terms of $\lambda$. Our 
result has to be read as follows: The initial configuration must be chosen such that its typical length scale is at least of order one, $1 \ll \frac{1}{E(m(0))}$, to ensure that we start within the interfacial regime, i.e., the mixture is separated into two domains of the two phases; but the typical length scale of the initial configuration is well below the crossover length, $L(m(0)) \ll 1$ (recall that for small length scales, $L$ is normalized by $\lambda^{1 / 2}$ ), cf. (3.4). The diffusive regime, $E \gg \frac{1}{\lambda^{1 / 2}}$ and $t \ll \lambda^{1 / 2}$, is only relevant if $\lambda \geq 1$. If so, the above result is a time-averaged version of $E \gtrsim \frac{1}{(\lambda t)^{1 / 3}}$, since in this situation $\max \left\{\lambda^{1 / 2} E^{2}, E\right\}=\lambda^{1 / 2} E^{2}$ and $\min \left\{\frac{\lambda^{1 / 2}}{(\lambda t)^{2 / 3}}, \frac{1}{t}\right\}=\frac{\lambda^{1 / 2}}{(\lambda t)^{2 / 3}}$. Likewise, in the regime $E \ll \frac{1}{\lambda^{1 / 2}}$ and $t \gg \lambda^{1 / 2}$, our result is a time-averaged version of $E \gtrsim \frac{1}{t}$.

We want to remark that upper bounds on coarsening rates are quite different from lower bounds. There are configurations, like parallel planar layers, that do not coarsen at all, or do so exponentially slowly. Therefore, lower bounds depend strongly on the initial data and can only be generically true.

The method we apply in our analysis was first introduced in [12. It exploits the gradient flow structure of the dynamics. In fact, the dynamical system (2.3) \& (2.4) (or analogously (2.7)-(2.9) ) can be interpreted as a gradient flow for the metric tensor

$$
\begin{aligned}
& g(\delta m, \delta m) \\
= & \inf _{j, u}\left\{f_{\Omega} \frac{1}{\lambda}|j|^{2} d x+f_{\Omega}|D u|^{2} d x \mid \delta m+\nabla \cdot(j+m u)=0, \nabla \cdot u=0\right\},
\end{aligned}
$$

cf. [4, Section 1.4]. The method of [12] now translates bounds on the energy landscape, i.e., information on how fast the energy can decrease as a function of a distance (originally, the distance induced by the metric tensor) to some reference configuration, to bounds on the dynamics, i.e., bounds on how fast the energy decreases as a function of time. The method consists mainly of three ingredients: an energy dissipation inequality, an interpolation (or isoperimetric) inequality, and an ODE argument.

The main contribution of this work is the dissipation inequality.

Proposition 4.3 (Dissipation inequality). Let $m$ be a solution of (2.4). Assume that $E(m(0)) \lesssim 1$. Then $L$ is an absolutely continuous function and for a.e. $t \geq 0$,

$$
\frac{d}{d t} L(t) \lesssim\left(f_{\Omega} \lambda|\operatorname{grad} \mu|^{2}+|D u|^{2} d x\right)^{1 / 2}
$$

The statement with $\lambda=1$ holds for the sharp interface system as well, again provided that it has regular enough solutions. We may also drop the condition on the initial energy.

The interpolation inequality we obtain holds for a very broad range of cost functions. It represents a diffuse-interface counterpart of the one proved in 4. One should note that the interpolation inequality for the sharp interface case does not require an assumption on energy smallness.

Proposition 4.4 (Interpolation inequality). Let $c$ be monotonically increasing with $c(0)=0$. Let $m \in L^{2}(\Omega)$ with $\bar{m}=0$. Assume $E(m) \ll 1$. Then there exists a constant $c_{0}$, dependent on the space dimension only, such that

$$
d_{c}\left(m_{+}, m_{-}\right) \gtrsim c\left(\frac{1}{c_{0} E(m)}\right) .
$$


Finally, we state the ODE argument. The inhomogeneous form of the time averages in the statement of Proposition 4.5 is due to the particular feature of the argument, which only allows for $L^{q}$ averages in time, with $1<q<3$ in the diffusive regime (for convenience, we choose $q=2$ ), and $q=1$ in the convective regime, in order to capture the explicit form of the cost function $c$ from the interpolation inequality in the particular regimes. Notice that in our formulation of the ODE argument below, we have absorbed $c_{0}$ and the constants from the inequalities in Propositions $4.3 \& 4.4$ into $E, L$, and the new constant $C$.

Proposition 4.5 (ODE argument). Let $E(t)$ and $L(t)$ be two absolutely continuous, nonnegative functions satisfying

$$
\frac{d L}{d t} \leq C\left(-\frac{d E}{d t}\right)^{1 / 2} \quad \text { for a.e. } t
$$

and

$$
L \geq \begin{cases}\frac{1}{\lambda^{1 / 2} E} & \text { if } 1 \leq \lambda^{1 / 2} E \\ 1+\ln \frac{1}{\lambda^{1 / 2} E} & \text { if } 1 \geq \lambda^{1 / 2} E .\end{cases}
$$

Assume that $2 L(0) \leq 1$. Then there is a constant $\tilde{C}$ such that

$$
\int_{0}^{T} \max \left\{\lambda^{1 / 2} E^{2}, E\right\} d t \geq \frac{1}{\tilde{C}} \int_{0}^{T} \min \left\{\lambda^{1 / 2} \frac{1}{(\lambda t)^{2 / 3}}, \frac{1}{t}\right\} d t,
$$

for any $T$ such that $\lambda^{-1 / 2} T \geq L(0)^{3}$.

Now we come back to the definition of $c$, (4.1), and the interpretation of $L$, (4.2), as a physical length scale. A natural choice of the physical length scale is related to the gradient flow structure of the dynamics: In a gradient flow, the metric tensor encodes the limiting dissipation mechanisms. In particular, the geodesic distance induced by the metric tensor measures the minimal amount of energy that is dissipated when "moving" from one point in configuration space to another. However, in our situation, the induced distance with metric tensor defined in (4.3) is not known explicitly. Therefore, we construct a proxy $L(m)$ that is bounded by the induced distance. The idea of approximating induced distance functions by MKR distances has been introduced in [16, 10].

We now motivate the choice of $c$. The cost function has to be chosen in such a way that the MKR distance is dominated by the induced distance function. In the purely diffusive setting, the induced distance reduces to the $H^{-1}$ norm. Though in this situation a proxy is not necessary, the authors of [12] use a slightly weaker norm, the $H^{-1, \infty}$ norm, which is equivalent to the Monge-Kantorovich distance (i.e., the MKR distance with cost function given by the Euclidean distance $c(z)=z)$, in order to treat simultaneously the case of surface diffusion (diffusion along the interfacial layer in contrast to diffusion through the bulk). In the purely convective setting, the induced distance is due to the viscous dissipation, i.e., $f_{\Omega}|\operatorname{grad} u|^{2} d x$. Since the dissipation mechanism only controls the gradient of the convecting velocity, it is not surprising that the cost functional in the MKR distance can only grow logarithmically: In the finite dimensional analogue, trajectories of a Lipschitz vector field can diverge exponentially fast. This idea is exploited in 44. Since our goal is to prove the crossover in the coarsening rates from diffusion- to convection-mediated coarsening, we combine the cost function from [12] and [4]: We use the Euclidean distance as cost function for 
distances smaller (measured in terms of $E$ ) than the crossover length, $\frac{1}{E} \sim \lambda^{1 / 2}$, cf. (3.4), and the logarithmic cost function for distances larger than the crossover length.

A key insight of our work is that combining the cost appropriately for the two problems corresponding to separate transport mechanisms works for the problem in which the transport mechanisms are combined.

We remark that there is a subtle but nontrivial difference from [4 in the definition of $L, d_{c}\left(m_{+}, m_{-}\right)$is used instead of $d_{c}(m+1,1)$. The quantity above is more natural for the problem considered. Furthermore the change is necessary since there are no known $L^{\infty}$ bounds on $m$ which are uniform in time (and which one could use to find $M$ such that $m+M \geq 0$ and then consider $\left.d_{c}(m+M, M)\right)$. However, the total integrals of $m_{+}$and $m_{-}$change in time, which needs to be accounted for when computing $\frac{d L}{d t}$ for example.

MKR distances have been successfully introduced as proxies for the induced distance function in the framework of [12] in two further situations: In [23], the author considers a Wasserstein distance (i.e., an MKR distance with cost function given by the square of the Euclidean distance) to prove an upper bound on the coarsening rates in a non-local, degenerate Cahn-Hilliard equation modeling biological aggregation and phase segregation in binary alloys. In [17, a model for viscous thin films is under consideration, in which droplet configurations coarsen by Ostwald ripening and droplet collision. The evolution is given by a Cahn-Hilliard-type equation, and the induced distance $i s$ the Wasserstein distance.

\section{Effect of material transport on optimal transportation distances}

We establish estimates on how much the optimal transportation distances between fluid components change if the fluid is perturbed. We consider two sources of perturbation: mass redistribution via a flux $j$ and bulk transport via a velocity vector field $u$. Since this situation is of general interest, in this section $j$ and $u$ are not assumed to be given by a model of demixing. Furthermore we do not assume the regularity of $j$ and $u$, so that the result applies to systems where only weak solutions are known to exist. More precisely, we consider $m$ to be a (distributional) solution of

$$
\partial_{t} m+\nabla \cdot j+\nabla \cdot(m u)=0
$$

on a torus $\Omega$, where $j$ and $u$ are given.

Effect of bulk transport on optimal transportation distances was considered in [4, Lemma 1]. We state the result below and refer to [4] for a proof.

Lemma 5.1. Assume that $c:[0, \infty) \longrightarrow[0, \infty)$ is smooth and nondecreasing, with $c(0)=$ 0 . We denote the associated cost function by the same letter: $c(x, y)=c(\|x-y\|)$, where $\|x-y\|$ denotes the distance on $\Omega$ considered as a torus. Let $u$ be a $C^{1}$ vector field with period cell $\Omega$. Let $m_{1}, m_{2} \in C\left(0, T ; L^{1}(\Omega)\right)$ be two distributional solutions of

$$
\partial_{t} m_{i}+\nabla \cdot\left(m_{i} u\right)=0
$$

such that $m_{1}(0)(\Omega)=m_{2}(0)(\Omega)$. Then

$$
\frac{\bar{d}^{+}}{d t} d_{c}\left(m_{1}(t), m_{2}(t)\right) \leq f f_{\Omega \times \Omega} c^{\prime}(\|x-y\|) \nabla_{x}\|x-y\| \cdot(u(x, t)-u(y, t)) d \pi(t),
$$

where $\pi(t)$ is an optimal transportation plan with cost $c$ between $m_{1}(t)$ and $m_{2}(t)$ and $\frac{\bar{d}^{ \pm}}{d t}$ is the one-sided upper derivative, i.e.,

$$
\frac{\bar{d}^{+}}{d t} f(t):=\limsup _{h \rightarrow 0+} \frac{f(t+h)-f(t)}{h} .
$$


The analogous result holds for the one-sided lower derivative.

When applying the above lemma to the demixing problems, we have in mind $m$ being the order parameter of average 0 and set $m_{1}=m_{+}$and $m_{2}=m_{-}$. The main difference between the situation considered in 4 and the one here is that the total mass of $m_{+}$, that is $m_{+}(t)(\Omega)$, is not constant in time in the diffuse-interface case. This is entirely due to the transport by flux $j$. The following lemma contains the essential estimate to handle that element. It is an integral version of the estimate on the rate of change of the transportation distance.

Lemma 5.2. Let $c:[0, \infty) \longrightarrow[0, \infty)$ be smooth and nondecreasing, with $c(0)=0$. Furthermore assume that $c$ is subadditive and that $c^{\prime}(0) \leq 1$. Let $m$ be an $L^{2}$ function on $\Omega$ and $j$ an $H^{1}$ vector field. Let $m^{\prime}=m+\nabla \cdot j$. Then

$$
d_{c}\left(m_{+}^{\prime}, m_{-}^{\prime}\right)-d_{c}\left(m_{+}, m_{-}\right) \leq f_{\Omega}|j| d x .
$$

Proof. Note that since $c$ is subadditive, nondecreasing, and $c(0)=0$, the function $c(x, y)=c(\|x-y\|)$ defines a metric on $\Omega$. This enables us to use Lemma A.1 in the Appendix to conclude

$$
d_{c}\left(m_{+}^{\prime}, m_{-}^{\prime}\right) \leq d_{c}\left(m_{+}^{\prime}+m_{-}, m_{-}^{\prime}+m_{+}\right)+d_{c}\left(m_{+}, m_{-}\right) .
$$

Observe that $d_{c}\left(m_{-}, m_{+}\right)=d_{c}\left(m_{+}, m_{-}\right)$. Let $d_{1}$ be the Monge-Kantorovich distance, that is the optimal transportation distance corresponding to the Euclidean distance cost. Since $c$ is subadditive and $c^{\prime}(0) \leq 1$, it holds that $c(z) \leq z$ for all $z \geq 0$. Therefore

$$
d_{c}\left(m_{+}^{\prime}+m_{-}, m_{-}^{\prime}+m_{+}\right) \leq d_{1}\left(m_{+}^{\prime}+m_{-}, m_{-}^{\prime}+m_{+}\right) .
$$

We now recall the following representation formula for $d_{1}$ (see [27, page 38]):

$$
d_{1}\left(m, m^{\prime}\right)=\inf \left\{f_{\Omega}|\tilde{j}| d x: \nabla \cdot \tilde{j}=m^{\prime}-m\right\} .
$$

On the one hand, this implies that

$$
d_{1}\left(m_{+}^{\prime}+m_{-}, m_{-}^{\prime}+m_{+}\right)=d_{1}\left(m_{+}-m_{-}, m_{+}^{\prime}-m_{-}^{\prime}\right)=d_{1}\left(m, m^{\prime}\right) .
$$

On the other hand, we have the estimate

$$
d_{1}\left(m, m^{\prime}\right) \leq f_{\Omega}|j| d x .
$$

Gathering the inequalities above we conclude that

$$
d_{c}\left(m_{+}^{\prime}, m_{-}^{\prime}\right) \leq f_{\Omega}|j| d x+d_{c}\left(m_{+}, m_{-}\right) .
$$

We now combine the results of Lemma 5.1 and Lemma 5.2 and generalize them to apply to less regular vector fields.

Lemma 5.3. Let $c:[0, \infty) \longrightarrow[0, \infty)$ be $C^{1}$ and concave and such that $c(0)=0$ and $c^{\prime}(0) \leq 1$. Assume that $u$ is vector field in $L^{1}\left(0, T ; L^{2}\left(\Omega, \mathbb{R}^{d}\right)\right)$ and $j$ is a vector field in $L^{1}\left(0, T ; L^{1}\left(\Omega, \mathbb{R}^{d}\right)\right)$. Let $m \in C\left(0, T ; L^{2}(\Omega)\right)$ be a distributional solution of

$$
\partial_{t} m+\nabla \cdot j+\nabla \cdot(m u)=0
$$


with $\bar{m}=0$. Let $L(t)=d_{c}\left(m_{+}(t), m_{-}(t)\right)$. Then for all $t \in[0, T)$ and $h \in(0, T-t)$,

$$
\begin{aligned}
& L(t+h)-L(t) \\
\leq & \int_{t}^{t+h} f_{\Omega}|j(x, s)| d x d s \\
& +\int_{t}^{t+h} f f_{\Omega \times \Omega} c^{\prime}(x-y) \nabla_{x}\|x-y\| \cdot(u(x, s)-u(y, s)) d \pi(s) d s,
\end{aligned}
$$

where $\pi(s)$ is an optimal transportation plan, for cost $c$, between $m_{+}(s)$ and $m_{-}(s)$.

From (5.2) and the assumptions on $j, u$, and $m$, it follows that $L$ is an absolutely continuous function. That allows us to use the (a.e.) derivative of $L$ in the subsequent calculations.

Proof.

Step 1. Assume that $u$ and $j$ are $C^{1}$ vector fields. To be able to separately account for the effects of the two transport mechanisms, we introduce for any $t \in[0, T)$ the solution $\rho_{t}$ of

$$
\begin{aligned}
\partial_{s} \rho_{t}+\nabla \cdot\left(\rho_{t} u\right) & =0 \quad \text { on } \Omega \times[t, T] \\
\rho_{t}(t) & =m(t) .
\end{aligned}
$$

Let $\sigma_{t}(s)=m(s)-\rho_{t}(s)$. When the subscript $t$ is clear from the context, we will omit it. To estimate the change of the transportation distance, we separate the contributions that come from the two transport mechanisms. In particular, by Lemma A.1 in the Appendix A and $\rho_{t}(t)=m(t)$, we have for $0<h<T-t$ and $\rho=\rho_{t}$,

$$
\begin{aligned}
& d_{c}\left(m_{+}(t+h), m_{-}(t+h)\right)-d_{c}\left(m_{+}(t), m_{-}(t)\right) \\
\leq & d_{c}\left(\rho_{+}(t+h), \rho_{-}(t+h)\right)-d_{c}\left(\rho_{+}(t), \rho_{-}(t)\right) \\
& +d_{c}\left(\rho_{+}(t+h)+m_{-}(t+h), \rho_{-}(t+h)+m_{+}(t+h)\right) .
\end{aligned}
$$

Now (5.2) follows from the two estimates

$$
\begin{aligned}
& d_{c}\left(\rho_{+}(t+h), \rho_{-}(t+h)\right)-d_{c}\left(\rho_{+}(t), \rho_{-}(t)\right) \\
\leq & \int_{t}^{t+h} f f_{\Omega \times \Omega} c^{\prime}(x-y) \nabla_{x}\|x-y\| \cdot(u(x, s)-u(y, s)) d \pi(s) d s
\end{aligned}
$$

and

$$
\begin{aligned}
& d_{c}\left(\rho_{+}(t+h)+m_{-}(t+h), \rho_{-}(t+h)+m_{+}(t+h)\right) \\
\leq & \int_{t}^{t+h} f_{\Omega}|j(x, s)| d x d s+o(h) .
\end{aligned}
$$

Indeed, dividing by $h$ and taking the limit $h \rightarrow 0$ establishes the upper bound for $\frac{\bar{d}^{+}}{d t} L(t)$ for every $t \in[0, T)$. Integrating in time then gives (5.2).

Obviously, by the definition of $\rho,(5.3)$ is a direct consequence of Lemma 5.1 We 
now argue in favor of (5.4). We estimate

$$
\begin{aligned}
& d_{c}\left(\rho_{+}(t+h)+m_{-}(t+h), \rho_{-}(t+h)+m_{+}(t+h)\right) \\
\leq & d_{1}\left(\rho_{+}(t+h)+m_{-}(t+h), \rho_{-}(t+h)+m_{+}(t+h)\right) \\
= & d_{1}(\rho(t+h), m(t+h)) \\
= & d_{1}(\sigma(t+h), 0) \\
\leq & \int_{t}^{t+h} f_{\Omega}|j(x, s)|+|\sigma(x, s) u(x, s)| d x d s,
\end{aligned}
$$

where we used $c(z) \leq z$, the representation of the $d_{1}$ distance given in (5.1), and the fact that $\sigma$ satisfies

$$
\partial_{s} \sigma+\nabla \cdot j+\nabla \cdot(\sigma u)=0
$$

with $\sigma(t)=0$. We have to show that the second term on the right hand side of the above inequality is of higher order. To do so, we multiply the equation by $\sigma$; integrating by parts gives for $s \geq t$ :

$$
\frac{d}{d s} \frac{1}{2}\|\sigma(s)\|_{L^{2}(\Omega)}^{2} \leq\|j(s)\|_{H^{1}(\Omega)}\|\sigma(s)\|_{L^{2}(\Omega)}+\frac{1}{2} f_{\Omega} \sigma(s)^{2}|\nabla \cdot u| d x .
$$

Above and in the rest of this proof, all the norms $\left(\|\cdot\|_{L^{2}(\Omega)},\|\cdot\|_{H^{1}(\Omega)}\right.$, etc.) are rescaled by the size of the domain. Dividing by $\|\sigma(s)\|_{L^{2}(\Omega)}$ yields

$$
\frac{d}{d s}\|\sigma(s)\|_{L^{2}(\Omega)} \leq C(j, u)\left(1+\|\sigma(s)\|_{L^{2}(\Omega)}\right)
$$

where $C=C(j, u)$ is a constant involving the $C^{1}$ bounds on $j$ and $u$. By Gronwall's inequality,

$$
\|\sigma(s)\|_{L^{2}(\Omega)} \leq \exp (C(s-t))-1
$$

for all $s \in[t, T]$. Therefore

$$
\int_{t}^{t+h} f_{\Omega}|\sigma(x, s) u(x, s)| d x d s=o(h) .
$$

Step 2. Consider $j \in L^{1}\left(0, T ; L^{1}(\Omega)\right)$, with $u$ still assumed to be $C^{1}$. Let $\eta_{\varepsilon}$ be a standard mollifier in space and time. Consider the interval $[t, t+h] \subset(0, T)$. Convolving with $\eta_{\varepsilon}$, where $\Omega$ is considered as a torus, gives that $m_{\varepsilon}:=m * \eta_{\varepsilon}$ is a solution of

$$
\partial_{t} m_{\varepsilon}+\nabla \cdot\left(m_{\varepsilon} u\right)+\nabla \cdot \tilde{j}_{\varepsilon}=0 \quad \text { on } \Omega \times[t, t+h],
$$

where $\tilde{j}_{\varepsilon}:=j * \eta_{\varepsilon}-m_{\varepsilon} u+(m u) * \eta_{\varepsilon}$. Note that $\tilde{j}_{\varepsilon}$ is $C^{1}$ and thus the assumptions of Step 1 are satisfied. Let for $s \in[t, t+h], L_{\varepsilon}(s):=d_{c}\left(m_{\varepsilon+}(s), m_{\varepsilon-}(s)\right)$, and let $\pi_{\varepsilon}(s)$ be an optimal transportation plan between $m_{\varepsilon+}(s)$ and $m_{\varepsilon-}(s)$. We have

$$
\begin{aligned}
& L_{\varepsilon}(t+h)-L_{\varepsilon}(t) \\
\leq & \int_{t}^{t+h} f f_{\Omega \times \Omega} c^{\prime}(x-y) \nabla_{x}\|x-y\| \cdot(u(x, s)-u(y, s)) d \pi_{\varepsilon}(s) d s \\
& +\int_{t}^{t+h} f_{\Omega}\left|\tilde{j}_{\varepsilon}(x, s)\right| d x d s .
\end{aligned}
$$


Since $m_{\varepsilon+}(s) \rightarrow m_{+}(s)$ and $m_{\varepsilon-}(s) \rightarrow m_{-}(s)$ in $L^{2}(\Omega)$ as $\varepsilon \rightarrow 0$, we conclude $L_{\varepsilon}(t) \rightarrow$ $L(t)$ and $L_{\varepsilon}(t+h) \rightarrow L(t+h)$ as $\varepsilon \rightarrow 0$.

Regarding the term involving the velocity $u$ : For any $s \in[t, t+h]$,

$$
f \int_{\Omega \times \Omega} c^{\prime}(x-y) \nabla_{x}\|x-y\| \cdot(u(x, s)-u(y, s)) d\left(\pi_{\varepsilon}(s)-\pi(s)\right) \rightarrow 0 \quad \text { as } \varepsilon \rightarrow 0,
$$

since $\pi_{\varepsilon}(s)-\pi(s) \rightarrow 0$ weakly in the sense of measures due to stability of optimal transportation plans (see Theorem 5.20 in [28]). Assumptions on $c$ imply that $0 \leq$ $c^{\prime}(z) \leq 1$ for all $z \geq 0$. Thus

$$
\begin{aligned}
& \left|f f_{\Omega \times \Omega} c^{\prime}(x-y) \nabla_{x}\|x-y\| \cdot(u(x, s)-u(y, s)) d\left(\pi_{\varepsilon}(s)-\pi(s)\right)\right| \\
\leq & f \int_{\Omega \times \Omega}|u(x, s)|+|u(y, s)| d\left(\pi_{\varepsilon}(s)+\pi(s)\right) \\
= & f_{\Omega}|u(x, s)|\left(m_{\varepsilon+}(x)+m_{+}(x)\right) d x+f_{\Omega}|u(y, s)|\left(m_{\varepsilon-}(y)+m_{+}(y)\right) d y,
\end{aligned}
$$

which enables us to use the dominated convergence theorem to obtain the integralin-time form of (5.5).

Since $\tilde{j}_{\varepsilon} \rightarrow j$ in $L^{1}\left(0, T ; L^{1}(\Omega)\right)$ as $\varepsilon \rightarrow 0$, convergence of the term involving $\tilde{j}_{\varepsilon}$ follows as well. So (5.2) follows in the $\varepsilon \rightarrow 0$ limit.

Step 3. Now consider $u \in L^{1}\left(0, T ; L^{2}(\Omega)\right)$ and $j \in L^{1}\left(0, T ; L^{1}(\Omega)\right)$. Again let $[t, t+$ $h] \subset(0, T)$. Let $\eta_{\varepsilon}$ be a mollifier in space and time and let $u_{\varepsilon}:=u * \eta_{\varepsilon}$. Then $m \in$ $C\left(0, T ; L^{2}(\Omega)\right)$ is a distributional solution of

$$
\partial_{t} m+\nabla \cdot\left(m u_{\varepsilon}\right)+\nabla \cdot\left(j+m\left(u-u_{\varepsilon}\right)\right)=0 \quad \text { on } \Omega \times[t, t+h] .
$$

Let $j_{\varepsilon}:=j+m\left(u-u_{\varepsilon}\right)$. Note that $j_{\varepsilon}, u_{\varepsilon}$ satisfy the assumption of Step 2. To take the limit $\varepsilon \rightarrow 0$ of

$$
\begin{aligned}
& L(t+h)-L(t) \\
\leq & \int_{t}^{t+h} f_{\Omega}\left|j_{\varepsilon}(x, s)\right| d x \\
& +\int f_{\Omega \times \Omega} c^{\prime}(x-y) \nabla_{x}\|x-y\| \cdot\left(u_{\varepsilon}(x, s)-u_{\varepsilon}(y, s)\right) d \pi(s) d s,
\end{aligned}
$$

it suffices to observe that $j_{\varepsilon} \rightarrow j$ in $L^{1}\left(0, T ; L^{1}(\Omega)\right)$ as $\varepsilon \rightarrow 0$,

$$
\begin{aligned}
& \mid f f_{\Omega \times \Omega} c^{\prime}(x-y) \nabla_{x}\|x-y\| \cdot\left(u_{\varepsilon}(x, s)-u(x, s)-\left(u_{\varepsilon}(y, s)-u(y, s)\right) d \pi(s) d s \mid\right. \\
\leq & f_{\Omega}\left|u_{\varepsilon}(x, s)-u(x, s)\right| m_{+}(x, s) d x+f_{\Omega}\left|u_{\varepsilon}(y, s)-u(y, s)\right| m_{-}(y, s) d y,
\end{aligned}
$$

integrate in time and use the fact that $u_{\varepsilon} \rightarrow u$ in $L^{1}\left(0, T ; L^{2}(\Omega)\right)$ and $m_{+}, m_{-} \in$ $L^{\infty}\left(0, T ; L^{2}(\Omega)\right)$.

For the case $t=0$, the result follows in the limit of the result on intervals $[\delta, \delta+h]$, due to continuity of both sides of (5.2) with respect to $t$. 


\section{Proof of the upper bound on coarsening rate}

6.1. Dissipation Inequality. We now turn to the proof of Proposition 4.3, It relies on the main result from the previous section, Lemma 5.3, which measures the effect of diffusive and convective material transport on transportation distances. Our proof follows closely the one of [4. Proposition 2.2], which in turn was inspired by estimates derived by Crippa \& DeLellis in [7, Theorem 2.1 resp. Theorem 3.3].

Proof. [Proof of Proposition 4.3 We apply Lemma 5.3 with $c$ replaced by $\lambda^{-1 / 2} c$. Then $c^{\prime}(0) \leq 1$ and $c^{\prime}(z) \leq \frac{1}{z}$, and we have for a.e. $t \geq 0$

$$
\frac{d}{d t} L(t) \leq f_{\Omega} \frac{1}{\lambda^{1 / 2}}|j| d x+f f_{\Omega \times \Omega} \frac{|u(x)-u(y)|}{|x-y|} d \pi,
$$

where $\pi$ is any optimal transport plan with respect to cost $c$ between $m_{+}(t)$ and $m_{-}(t)$. Recall that $j=-\lambda \nabla \mu$ in our setting. Thus

$$
f_{\Omega} \frac{1}{\lambda^{1 / 2}}|j| d x \leq\left(f_{\Omega} \lambda|\nabla \mu|^{2} d x\right)^{\frac{1}{2}}
$$

The estimate for the contribution coming from convective transport is exactly the one from [4, Proposition 2]. For the convenience of the reader, we repeat the argument. The proof is based on maximal functions, $M(f)(x)=\sup _{r>0} \frac{1}{\left|B_{r}\right|} \int_{B_{r}(x)}|f| d x$, cf. [24]. Maximal functions have the following basic properties:

$$
|f(x)-f(y)| \lesssim(M(D f)(x)+M(D f)(y))|x-y|,
$$

and

$$
f_{\Omega}|M f|^{2} d x \lesssim f_{\Omega}|f|^{2} d x .
$$

Using these properties of $M f$, we have

$$
\begin{aligned}
& f f_{\Omega \times \Omega} \frac{|u(x)-u(y)|}{|x-y|} d \pi \\
\lesssim & f f_{\Omega \times \Omega}(M(D f)(x)+M(D f)(y)) d \pi \\
\lesssim & f_{\Omega} M(D u)\left(m_{+}+m_{-}\right) d x \\
\lesssim & \left(f_{\Omega} M(D u)^{2} d x\right)^{\frac{1}{2}}\left(f_{\Omega} m^{2} d x\right)^{\frac{1}{2}} \\
\lesssim & \left(f_{\Omega}|D u|^{2} d x\right)^{\frac{1}{2}} .
\end{aligned}
$$

Above we used that

$$
\begin{aligned}
f_{\Omega} m^{2} d x & =f_{\{|m|>2\}} m^{2} d x+f_{\{|m| \leq 2\}} m^{2} d x \\
& \leq f_{\{|m|>2\}}\left(1-m^{2}\right)^{2} d x+f_{\{|m| \leq 2\}} 2^{2} d x \\
& \leq E+4 \lesssim 5,
\end{aligned}
$$


since $E \leq E(m(0)) \lesssim 1$. Combining the estimates above and the expression for the energy dissipation, (2.5), gives the desired estimate.

For the sharp-interface model, we no longer assume that $E \lesssim 1$. Also recall that we take $\lambda=1$. The proof is entirely analogous to the diffuse-interface case, so we do not present the details.

6.2. Interpolation Inequality. The interpolation inequality for sharpinterfaces is the one proved in [4, Proposition 3]. The one for diffuse interfaces can be proved similarly. It requires the additional result that the domain is essentially divided into phases. We present a proof below for completeness. We also remark that an alternative proof of the inequality follows from the proof of Theorem 6 (and Corollary 7) in [23] (in particular Step 5 and Step 6 imply that in any coupling between $m_{+}$and $m_{-}$a significant proportion of the mass has to be transported over distances at least of size $1 / E$, which implies the inequality). Recall that the interpolation inequality was stated in Proposition 4.4 .

Proof. [Proof of Proposition 4.4] We introduce some notations. We denote by $A$ the set essentially occupied by one phase, and by $\chi$ its indicator function. More precisely,

$$
A=\left\{x \in \Omega: m(x) \geq \frac{1}{2}\right\} \quad \text { and } \quad \chi=\chi_{A} .
$$

Furthermore, let $h_{W}$ denote some constant satisfying

$$
W(m) \geq \begin{cases}2 h_{W}(|m|-1) & \text { for }|m| \geq \frac{3}{2}, \\ h_{W} & \text { for }|m| \leq \frac{1}{2} .\end{cases}
$$

Here, $W(m)$ denotes the potential energy, $W(m):=\frac{1}{2}\left(1-m^{2}\right)^{2}$. (It is easy to check that $h_{W}=\frac{9}{32}$ is optimal.) We first claim that

$$
\|A\|:=\frac{|A \cap \Omega|}{|\Omega|} \gtrsim 1
$$

We split the phase space according to

$$
\begin{aligned}
1= & f_{\left\{m \leq-\frac{1}{2}\right\}}(m+1) d x+f_{\left\{-\frac{1}{2}<m<\frac{1}{2}\right\}}(m+1) d x \\
& +f_{\left\{\frac{1}{2} \leq m \leq \frac{3}{2}\right\}}(m+1) d x+f_{\left\{\frac{3}{2}<m\right\}}(m+1) d x \\
\leq & \frac{1}{2}+\frac{3}{2} f_{\left\{-\frac{1}{2}<m<\frac{1}{2}\right\}} 1 d x+\frac{5}{2}\|A\|+f_{\left\{\frac{3}{2}<m\right\}}(m-1) d x+2\left\|\left\{\frac{3}{2}<m\right\}\right\| \\
\leq & \frac{1}{2}+\frac{3}{2 h_{W}} f_{\Omega} W(m) d x+\frac{5}{2}\|A\|+\frac{1}{2 h_{W}} f_{\Omega} W(m) d x+2\|A\| \\
\leq & \frac{1}{2}+\frac{2}{h_{W}} f_{\Omega} W(m) d x+\frac{9}{2}\|A\| .
\end{aligned}
$$

We can rewrite the estimate as $1 \leq \frac{4}{h_{W}} E+9\|A\|$. Due to the assumption $E(m) \ll 1$, this implies (6.1). 
In the sequel, the superscript $R$ denotes the convolution with a standard mollifier supported in the ball of radius $R$. From (6.1) and the definition of $A$ we find

$$
1 \lesssim f_{\Omega} \chi d x \lesssim f_{\Omega} \chi m d x
$$

which we split according to

$$
1 \lesssim f_{\Omega} \chi\left(m-m^{R}\right) d x+f_{\Omega} \chi m^{R} d x
$$

For the first term in (6.2), we proceed as in [12. Defining

$$
U(m):=\int_{0}^{m}\left|1-\tilde{m}^{2}\right| d \tilde{m}
$$

we have $\frac{d U}{d m}=\left|1-m^{2}\right|$, and thus

$$
\begin{aligned}
f_{\Omega}|\nabla(U(m))| d x & =f_{\Omega}|\nabla m| \frac{d U}{d m} d x \\
& \leq f_{\Omega} \frac{1}{2}|\nabla m|^{2}+\frac{1}{2}\left(\frac{d U}{d m}\right) d x=E .
\end{aligned}
$$

On the other hand, by the definition of $U(m)$, it holds $\left(m_{1}-m_{2}\right)^{2} \lesssim\left|U\left(m_{1}\right)-U\left(m_{2}\right)\right|$, so that

$$
\begin{aligned}
\left|f_{\Omega} \chi\left(m-m^{R}\right) d x\right| & \lesssim \frac{1}{\delta} f_{\Omega}\left(m-m^{R}\right)^{2} d x+\delta f_{\Omega} \chi^{2} d x \\
& \leq \frac{1}{\delta} \sup _{|y| \leq R} f_{\Omega}(m(x)-m(x+y))^{2} d x+\delta \\
& \lesssim \frac{1}{\delta} \sup _{|y| \leq R} f_{\Omega}|U(m(x))-U(m(x+y))| d x+\delta \\
& \lesssim \frac{R}{\delta} f_{\Omega}|\nabla(U(m))| d x+\delta .
\end{aligned}
$$

Thanks to the Modica-Mortola estimate (6.3), we obtain

$$
\left|f_{\Omega} \chi\left(m-m^{R}\right) d x\right| \lesssim \frac{R}{\delta} E+\delta .
$$

For the second term in (6.2), we argue as in (4). We have, for any admissible transfer plan $\pi$,

$$
\begin{aligned}
f_{\Omega} \chi m^{R} d x & =f_{\Omega} \chi^{R} m d x \\
& =f_{\Omega} \chi^{R} m_{+} d x-f_{\Omega} \chi^{R} m_{-} d x \\
& =f f_{\Omega \times \Omega}\left(\chi^{R}(x)-\chi^{R}(y)\right) d \pi(x, y) .
\end{aligned}
$$


To estimate the last term, we must deal separately with transport over large and small distances:

$$
\begin{aligned}
\left|f_{\Omega} \chi m^{R} d x\right| \leq & f f_{\{|x-y| \leq r\}}\left|\chi^{R}(x)-\chi^{R}(y)\right| d \pi(x, y) \\
& +f f_{\{|x-y|<r\}}\left|\chi^{R}(x)-\chi^{R}(y)\right| d \pi(x, y) .
\end{aligned}
$$

For the small distances, we find

$$
\begin{aligned}
&\left|f f_{\{|x-y| \leq r\}}\right| \chi^{R}(x)-\chi^{R}(y)|d \pi(x, y)| \\
& \leq \sup \left|\nabla \chi^{R}\right| f f_{\{|x-y| \leq r\}}|x-y| d \pi(x, y) \lesssim \frac{r}{R} .
\end{aligned}
$$

For large distances, we use the monotonicity of $c$ :

$$
\begin{aligned}
& \left|f f_{\{|x-y|>r\}}\right| \chi^{R}(x)-\chi^{R}(y)|d \pi(x, y)| \\
\leq & 2 \sup \left|\chi^{R}\right| \frac{1}{c(r)} \int f_{\Omega \times \Omega} c(|x-y|) d \pi(x, y) \lesssim \frac{d_{c}\left(m_{+}, m_{-}\right)}{c(r)} .
\end{aligned}
$$

Substituting (6.6) and (6.7) in (6.5) we conclude

$$
\left|f_{\Omega} \chi m^{R} d x\right| \lesssim \frac{r}{R}+\frac{d_{c}\left(m_{+}, m_{-}\right)}{c(r)}
$$

In view of (6.4) and (6.8), inequality (6.2) turns into

$$
1 \lesssim \frac{R E}{\delta}+\frac{r}{R}+\frac{d_{c}\left(m_{+}, m_{-}\right)}{c(r)}+\delta
$$

Choosing $\delta$ sufficiently small and optimizing in $R$ yields

$$
2\left(\frac{1}{c_{0}}\right)^{1 / 2} \leq(r E)^{1 / 2}+\frac{d_{c}\left(m_{+}, m_{-}\right)}{c(r)},
$$

when we reintroduce some constant $c_{0}$. Choosing $r=\frac{1}{c_{0} E}$, this becomes

$$
\left(\frac{1}{c_{0}}\right)^{1 / 2} \leq \frac{d_{c}\left(m_{+}, m_{-}\right)}{c\left(\frac{1}{c_{0} E}\right)}
$$

which is the desired estimate of Proposition 4.4.

\section{5 .}

6.3. ODE argument. In this section, we provide the proof of Proposition

Proof. [Proof of Proposition 4.5] By rescaling

$$
E=\lambda^{-1 / 2} \hat{E}, \quad L=\hat{L}, \quad t=\lambda^{1 / 2} \hat{t},
$$


we may without loss of generality assume that $\lambda=1$. We set for abbreviation:

$$
c(s):=\left\{\begin{array}{ll}
s & \text { for } s \leq 1 \\
1+\ln s & \text { for } s \geq 1
\end{array}\right\},
$$

(which is our cost function in the definition of $L$ with $\lambda=1$,)

$$
f(e):=\left\{\begin{array}{l}
e \text { for } e \leq 1 \\
e^{2} \text { for } e \geq 1
\end{array}\right\}=\max \left\{e^{2}, e\right\}
$$

and

$$
g(e):=c\left(\frac{1}{e}\right)=\left\{\begin{array}{ll}
1+\ln \frac{1}{e} & \text { for } e \leq 1 \\
\frac{1}{e} & \text { for } e \geq 1
\end{array}\right\}
$$

Notice that $g$ and $f$ are related by

$$
g^{\prime}(e)=\left\{\begin{array}{l}
-\frac{1}{e} \text { for } e \leq 1 \\
-\frac{1}{e^{2}} \text { for } e \geq 1
\end{array}\right\}=-\frac{1}{f(e)}
$$

Hence, as long as $L(T) \geq 2 L(0)$, we have

$$
L(T) \leq 2(L(T)-L(0))=2 \int_{0}^{T} \frac{d L}{d t} d t,
$$

and thus, applying (4.4), 4.5), and the definition of $g$,

$$
g(E(T)) \leq \tilde{C} \int_{0}^{T}\left(-\frac{d E}{d t}\right)^{1 / 2} d t
$$

where $\tilde{C}$ denotes a generic constant whose value may change from line to line. Without loss of generality, we may assume that $\tilde{C} \geq 1$. With the help of the Cauchy-Schwarz inequality, this estimate turns into

$$
g(E(T)) \leq \tilde{C}\left(\int_{0}^{T} \frac{1}{f(E)}\left(-\frac{d E}{d t}\right) d t \int_{0}^{T} f(E) d t\right)^{1 / 2}
$$

By the relation between $f$ and $g$, it is

$$
\frac{1}{f(E)}\left(-\frac{d E}{d t}\right)=g^{\prime}(E) \frac{d E}{d t}=\frac{d}{d t} g(E)
$$

and thus

$$
\int_{0}^{T} \frac{1}{f(E)}\left(-\frac{d E}{d t}\right) d t=g(E(T))-g(E(0)) \leq g(E(T)),
$$

so that (6.9) implies

$$
g(E(T)) \leq \tilde{C}\left(g(E(T)) \int_{0}^{T} f(E) d t\right)^{1 / 2}
$$


or equivalently

$$
g(E(T)) \leq \tilde{C} \int_{0}^{T} f(E) d t
$$

We rewrite this estimate as an implicit ODE for $h(T):=\int_{0}^{T} f(E) d t$ :

$$
\left(g \circ f^{-1}\right)\left(h^{\prime}(T)\right) \leq \tilde{C} h(T) .
$$

Since $g$ is a decreasing and $f^{-1}$ an increasing function, the above inequality is equivalent to

$$
h^{\prime}(T) \geq\left(f \circ g^{-1}\right)(\tilde{C} h(T)) .
$$

Let $F$ be the antiderivative of $\frac{1}{f \circ g^{-1}}$ with $F(0)=0$. An easy calculation shows

$$
F(z)=\left\{\begin{array}{ll}
\frac{1}{3} z^{3} & \text { for } z \leq 1 \\
\exp (z-1)-\frac{2}{3} & \text { for } z \geq 1
\end{array}\right\}
$$

Then the above inequality can be rewritten as

$$
\frac{d}{d T} F(\tilde{C} h(T)) \geq \tilde{C}
$$

which turns into

$$
F(\tilde{C} h(T))=F(\tilde{C} h(T))-F(\tilde{C} h(0)) \geq \tilde{C} T \stackrel{\tilde{C} \geq 1}{\geq} T
$$

after integration. Since $F$ is an increasing function, this can be paraphrased as

$$
\begin{aligned}
\tilde{C} h(T) & \geq F^{-1}(T) \\
& =\left\{\begin{array}{ll}
(3 T)^{1 / 3} & \text { for } T \leq \frac{1}{3} \\
1+\ln \left(T+\frac{2}{3}\right) & \text { for } T \geq \frac{1}{3}
\end{array}\right\} \\
& \sim \int_{0}^{T} \min \left\{\left(\frac{1}{t^{1 / 3}}\right)^{2}, \frac{1}{t}\right\} d t .
\end{aligned}
$$

By the definition of $h$, this turns into

$$
\int_{0}^{T} \max \left\{E^{2}, E\right\} d t \geq \frac{1}{\tilde{C}} \int_{0}^{T} \min \left\{\left(\frac{1}{t^{1 / 3}}\right)^{2}, \frac{1}{t}\right\} d t .
$$

We now argue for the case that $L(T) \leq 2 L(0)$. By (4.5), we have

$$
c\left(\frac{1}{E(T)}\right) \leq L(T) \leq 2 L(0) \text {. }
$$

Since $2 L(0) \leq 1$, it is $c\left(\frac{1}{E(T)}\right) \leq 1$, and thus $c\left(\frac{1}{E(T)}\right)=\frac{1}{E(T)}$. Consequently, the above estimate can be rewritten as

$$
\frac{1}{E(T)} \leq L(T) \leq 2 L(0)
$$


Since $L(0) \leq T^{1 / 3}$, it is

$$
\frac{1}{E(T)} \leq 2 T^{1 / 3}
$$

and since $E(t)$ is an decreasing function, this implies

$$
E(t) \geq E(T) \geq \frac{1}{2 T^{1 / 3}},
$$

for all $0 \leq t \leq T$. We square both sides and integrate in time:

$$
\begin{aligned}
\int_{0}^{T} \max \left\{E^{2}, E\right\} d t & \geq \int_{0}^{T} E^{2} d t \\
& \geq \frac{1}{4} T^{1 / 3} \\
& =\frac{1}{12} \int_{0}^{T}\left(\frac{1}{t^{1 / 3}}\right)^{2} d t \\
& \geq \frac{1}{12} \int_{0}^{T} \min \left\{\left(\frac{1}{t^{1 / 3}}\right)^{2}, \frac{1}{t}\right\} d t
\end{aligned}
$$

It remains to combine (6.10) and (6.11) to conclude the proof of Proposition 4.5,

Acknowledgments. CS thanks Helmut Abels for discussions and Carnegie Mellon University for hospitality. He was supported by the German Science Foundation through SFB 611. DS was supported by NSF grants DMS-0638481 and DMS-0908415. $\mathrm{He}$ is thankful to the University of Bonn and MPI Leipzig for hospitality. He is also grateful to the Center for Nonlinear Analysis (NSF grant DMS-0635983) for its support. The research collaboration was also supported by NSF PIRE grant OISE0967140.

Appendix A. Here we establish some properties of optimal transportation distances.

Lemma A.1. Let $c: \Omega \times \Omega \longrightarrow[0, \infty)$ be a metric on $\Omega$. Then for all positive measures $\theta_{1}, \theta_{2}, \vartheta_{1}$, and $\vartheta_{2}$, such that $\theta_{1}(\Omega)=\vartheta_{1}(\Omega)$ and $\theta_{2}(\Omega)=\vartheta_{2}(\Omega)$,

$$
d_{c}\left(\theta_{1}, \vartheta_{1}\right) \leq d_{c}\left(\theta_{1}+\theta_{2}, \vartheta_{1}+\vartheta_{2}\right)+d_{c}\left(\theta_{2}, \vartheta_{2}\right) .
$$

Proof. By Kantorovich duality, cf. [27, Theorem 1.3],

$$
d_{c}\left(\theta_{1}, \vartheta_{1}\right)=\sup _{\{\phi, \psi \mid \phi(x)+\psi(y) \leq c(x, y)\}} f_{\Omega} \phi d \theta_{1}+f_{\Omega} \psi d \vartheta_{1} .
$$

Furthermore it is enough to consider pairs such that $\phi$ and $\psi$ are $c$-duals of each other, for instance

$$
\psi=\phi^{c} \text { and } \phi=\psi^{c},
$$

where the $c$-duals are defined as

$$
\phi^{c}(y)=\inf _{x \in \Omega}\{c(x, y)-\phi(x)\} \quad \text { and } \quad \psi^{c}(x)=\inf _{y \in \Omega}\{c(x, y)-\psi(y)\} .
$$


We claim that if $\phi=\psi^{c}$ for some $\psi$ then for all $x_{1}, x_{2} \in \Omega$,

$$
\left|\phi\left(x_{1}\right)-\phi\left(x_{2}\right)\right| \leq c\left(x_{1}, x_{2}\right) .
$$

To see this, note that by the definition of $\psi^{c}$, for any $\varepsilon>0$ and $x_{1}, x_{2} \in \Omega$ there exists $x_{1}^{\prime}$ such that

$$
\phi\left(x_{1}\right) \geq c\left(x_{1}, x_{1}^{\prime}\right)-\psi\left(x_{1}^{\prime}\right)-\varepsilon .
$$

Therefore

$$
\phi\left(x_{2}\right) \leq c\left(x_{2}, x_{1}^{\prime}\right)-\psi\left(x_{1}^{\prime}\right) \leq c\left(x_{2}, x_{1}^{\prime}\right)-c\left(x_{1}^{\prime}, x_{1}\right)+\phi\left(x_{1}\right)+\varepsilon \leq c\left(x_{1}, x_{2}\right)+\phi\left(x_{1}\right)+\varepsilon .
$$

Letting $\varepsilon \rightarrow 0$ and using symmetry in $x_{1}, x_{2}$ allows us to conclude (A.1). By similar arguments, based on (A.1), we prove that

$$
-\phi(x)-\psi(y) \leq c(x, y) .
$$

We deduce that

$$
\begin{aligned}
& f_{\Omega} \phi d \theta_{1}+f_{\Omega} \psi d \vartheta_{1} \\
= & f_{\Omega} \phi d\left(\theta_{1}+\theta_{2}\right)+f_{\Omega} \psi d\left(\vartheta_{1}+\vartheta_{2}\right)-\left(f_{\Omega} \phi d \theta_{2}+f_{\Omega} \psi d \vartheta_{2}\right) \\
\leq & d_{c}\left(\theta_{1}+\theta_{2}, \vartheta_{1}+\vartheta_{2}\right)+f f_{\Omega \times \Omega} c(x, y) d \pi_{2}(x, y) .
\end{aligned}
$$

It remains to optimize in $\phi, \psi$, and $\pi_{2}$.

\section{REFERENCES}

[1] H. Abels and M. Röger, Existence of weak solutions for a non-classical sharp interface model for a two-phase flow of viscous, incompressible fluids, Ann. Inst. H. Poincaré Anal. Non Linéaire, 26(6), 2403-2424, 2009.

[2] F.J. Alexander, S. Chen, and D.W. Grunau, Hydrodynamic spinodal decomposition: Growth kinetics and scaling functions, Phys. Rev. B, 48(1), 634-637, 1993.

[3] N.D. Alikakos, P.W. Bates, and X. Chen, Convergence of the Cahn-Hilliard equation to the Hele-Shaw model, Arch. Rational Mech. Anal., 128(2), 165-205, 1994.

[4] Y. Brenier, F. Otto, and C. Seis, Upper bounds on coarsening rates in demixing binary viscous liquids, SIAM J. Math. Anal., 43(1), 114-134, 2011.

[5] X. Chen, Global asymptotic limit of solutions of the Cahn-Hilliard equation, J. Diff. Geom., 44(2), 262-311, 1996.

[6] Y.C. Chou and W.I. Goldburg, Phase separation and coalescence in critically quenched isobutyric-acid-water and 2,6-lutidine-water mixtures, Phys. Rev. A, 20(5), 2105-2113, 1979.

[7] G. Crippa and C. De Lellis, Estimates and regularity results for the DiPerna-Lions flow, J. Reine Angew. Math., 616, 15-46, 2008.

[8] S. Dai, B. Niethammer, and R.L. Pego, Crossover in coarsening rates for the monopole approximation of the Mullins-Sekerka model with kinetic drag, Proc. Roy. Soc. Edinburgh Sect. A, 140(3), 553-571, 2010.

[9] H. Furukawa, Spinodal decomposition of two-dimensional fluid mixtures: A spectral analysis of droplet growth, Phys. Rev. E, 61(2), 1423-1431, 2000.

[10] R. Jordan, D. Kinderlehrer, and F. Otto, The variational formulation of the Fokker-Planck equation, SIAM J. Math. Anal., 29(1), 1-17, 1998.

[11] T. Koga and K. Kawasaki, Late stage dynamics of spinodal decomposition in binary fluid mixtures, Phys. Stat. Mech. Appl., 196, 389-415, 1993. 
[12] R.V. Kohn and F. Otto, Upper bounds on coarsening rates, Commun. Math. Phys., 229(3), 375-395, 2002.

[13] K. Kubota, N. Kuwahara, H. Eda, M. Sakazume, and K. Takiwaki, Dynamic scaling behavior of spinodal decomposition in a critical mixture of 2,5-hexanediol and benzene, J. Chem. Phys., 97(12), 9291-9298, 1992.

[14] I.M. Lifshitz and V.V. Slyozov, The kinetics of precipitation from supersaturated solid solutions, J. Phys. Chem. Solids, 19(1-2), 35-50, 1961.

[15] T. Lookman, Y. Wu, F.J. Alexander, and S. Chen, Spinodal decomposition in fluids: Diffusive, viscous, and inertial regimes, Phys. Rev. E, 53(5), 5513-5516, 1996.

[16] F. Otto, The geometry of dissipative evolution equations: The porous medium equation, Commun. Part. Diff. Eqs., 26(1-2), 101-174, 2001.

[17] F. Otto, T. Rump, and D. Slepčev, Coarsening rates for a droplet model: Rigorous upper bounds, SIAM J. Math. Anal., 38(2), 503-529, (electronic) 2006.

[18] F. Otto, C. Seis, and D. Slepčev, Crossover of the coarsening rates in demixing of binary viscous liquids, CNA preprint, http://www.math.cmu.edu/CNA/, MPI preprint, http://www.mis.mpg.de/publications/preprints/2012/prepr2012-21.html, 2012.

[19] R.L. Pego, Front migration in the nonlinear Cahn-Hilliard equation, Proc. Roy. Soc. London Ser. A, 422(1863), 261-278, 1989.

[20] S. Puri and B. Dünweg, Temporally linear domain growth in the segregation of binary fluids, Phys. Rev. A, 45(10), R6977-R6980, 1992.

[21] M. San Miguel, M. Grant, and J.D. Gunton, Phase separation in two-dimensional binary fluids, Phys. Rev. A, 31(2), 1001-1005, 1985.

[22] E.D. Siggia, Late stages of spinodal decomposition in binary mixtures, Phys. Rev. A, 20(2), 595-605, 1979

[23] D. Slepčev, Coarsening in nonlocal interfacial systems, SIAM J. Math. Anal., 40(3), 1029-1048, 2008.

[24] E.M. Stein, Singular Integrals and Differentiability Properties of Functions, Princeton Mathematical Series, No. 30. Princeton University Press, Princeton, N.J., 1970.

[25] O.T. Valls and J.E. Farrell, Spinodal decomposition in a three-dimensional fluid model, Phys. Rev. E, 47(1), R36-R39, 1993.

[26] O.T. Valls and G.F. Mazenko, Growth kinetics for a model two-dimensional fluid, Phys. Rev. B, 38(16), 11643-11649, 1988.

[27] C. Villani, Topics in Optimal Transportation, Graduate Studies in Mathematics, American Mathematical Society, Providence, RI, 58, 2003.

[28] C. Villani, Optimal Transport: Old and New, in Grundlehren der Mathematischen Wissenschaften [Fundamental Principles of Mathematical Sciences], Springer-Verlag, Berlin, 338,2009 .

[29] C. Wagner, Theorie der Alterung von Niederschlägen durch Umlösen (Ostwald-Reifung), Zeitschrift für Elektrochemie, Berichte der Bunsengesellschaft für physikalische Chemie, 65(7-8), 581-591, 1961.

[30] N.-C. Wong and C.M. Knobler, Light scattering studies of phase separation in isobutyric acid + water mixtures, J. Chem. Phys., 69, 725-735, 1978.

[31] N.-C. Wong and C.M. Knobler, Light-scattering studies of phase separation in isobutyric acid + water mixtures: Hydrodynamic effects, Phys. Rev. A, 24(6), 3205-3211, 1981. 